\title{
Graph Models of Critical Infrastructure Interdependencies
}

\author{
Nils Kalstad Svendsen ${ }^{1}$ and Stephen D. Wolthusen ${ }^{1,2}$ \\ ${ }^{1}$ Norwegian Information Security Laboratory, Gjøvik University College, \\ P.O. Box 191, N-2802 Gjøvik, Norway \\ ${ }^{2}$ Information Security Group, Department of Mathematics, Royal Holloway, \\ University of London, Egham Hill, Egham TW20 0EX, UK
}

\begin{abstract}
Critical infrastructures are interconnected on multiple levels, and due to their size models with acceptable computational complexity and adequate modeling capacities must be developed. This paper presents the skeleton of a graph based model and sketches its capabilities.
\end{abstract}

\section{Introduction}

Critical infrastructures, including primarily the energy, financial services, health care, public services, and transportation sectors [12], are interconnected and interdependent on multiple levels. This leads to a number of questions which must be answered satisfactorily to protect the well-being of the population, functioning of government, and economic capabilities. Questions may include what cascading effects a regional failure of one critical infrastructure (such as the recent November 2006 failure of the electric power grid throughout much of continental Europe [3] and the August 2003 power outages in the northeastern U.S. and Canada 4]) may have on other infrastructure components, or to elaborate how adding small and hence cost-effective amounts of redundancy can significantly enhance the overall robustness of this interconnected network of infrastructure services.

While elaborate models exist for many individual infrastructures, it is desirable to also investigate larger-scale interactions among multiple infrastructure sectors. Research questions include the conditions for cascading effects resulting from isolated and coordinated infrastructure component failures, together with circular and transitive effects that might inhibit or at least severely impede the resumption of regular infrastructure services. This requires the development of models of acceptable computational complexity providing adequate modeling capabilities. The level of detail which can be incorporated in such models is limited compared to sector-specific models; however, in many cases the basic identification of interdependencies and critical dependency paths between infrastructure components already provides valuable information.

We describe a general graph-theoretical modeling and analysis framework based on multigraphs which can be used to analyze simple connectivity models, but which is also extensible to characterize particular types and interdependencies in more detail. The graph-theoretical model provides a set of efficient and 
well-understood formalisms also amenable to algorithmic investigation that a less rigorously formulated approach (e.g. agent-based simulations) cannot provide. Examples of possible model extensions include domain-specific abstract models which take the properties of certain types of infrastructures (e.g. for the electric power grid, pipelines, or even command and control structures) into account and use this family of models to analyze interdependencies among multiple types of infrastructures. Of particular interest to our research are issues involving transitive and circular interdependencies which may not be immediately obvious, may incorporate feedback and amplification, or even ringing and time-dependencies within the infrastructure network. Results from such analysis can e.g. help to devise more robust critical infrastructure networks or, in case of emergencies and disasters, help to prioritize resources to maintain minimum levels of service or to prevent the collapse of infrastructure webs.

\section{Model Overview}

This section gives an overview of our proposed model. For further details and discussions on its interrelationship with other models we refer to [5]67].

Interactions among infrastructure components and infrastructure users are modeled in the form of directed multigraphs, further augmented by response functions defining interactions between components. In the model, the vertices $\mathcal{V}=\left\{v_{1}, \ldots, v_{k}\right\}$ are interpreted as producers and consumers of $m$ different types of services or dependency types chosen from the set $\mathcal{D}=\left\{d_{1}, \ldots, d_{m}\right\}$. It is assumed that all nodes $v_{a}$ have a buffer of volume $V_{a}^{j}$ for all dependency types $d_{j}$. Each node also has a capacity limit $N_{\operatorname{Max}}\left(v_{a}, d_{j}\right)$ in terms of the amount of resource $d_{j}$ that can be stored in the node. The dependency types can be classified as ephemeral ( $V_{a}^{j}=0$ for all nodes $v_{a}$, and it follows that $\left.N_{\operatorname{Max}}\left(v_{a}, d_{j}\right)=0\right)$, storeable and incompressible $\left(N_{\operatorname{Max}}\left(v_{a}, d_{j}\right)=\rho V_{a}, \rho\right.$ is the density of the resource), or storeable and compressible $\left(N_{\mathrm{Max}}\left(v_{a}, d_{j}\right)=\right.$ $P_{\text {Max }}\left(v_{a}, d_{j}\right) V_{a}, P_{\text {Max }}\left(v_{a}, d_{j}\right)$ is the maximum pressure supported in the storage of resource $d_{j}$ in the node $v_{a}$ ). Pairwise dependencies between nodes are represented with directed edges, where the head node is dependent on the tail node. The edges of a given infrastructure are defined by a subset $\mathcal{E}$ of $\mathcal{E}=\left\{e_{1}^{1}, e_{2}^{1}, \ldots, e_{n_{1}}^{1}, e_{1}^{2}, \ldots, e_{n_{m}}^{m}\right\}$, where $n_{1}, \ldots, n_{m}$ respectively are the numbers of dependencies of type $d_{1}, \ldots, d_{m}$, and $e_{i}^{j}$ is the edge number $i$ of dependency type $j$ in the network. A given dependency between two nodes $v_{a}$ and $v_{b}$ is uniquely determined by $e_{i}^{j}\left(v_{a}, v_{b}\right)$. Further, two predicates $C_{\mathrm{Max}}\left(e_{i}^{j}\left(v_{a}, v_{b}\right)\right) \in \mathbb{N}_{0}$ and $C_{\text {Min }}\left(e_{i}^{j}\left(v_{a}, v_{b}\right)\right) \in \mathbb{N}_{0}$ are defined for each edge. These values respectively represent the maximum capacity of the edge $e_{i}^{j}\left(v_{a}, v_{b}\right)$ and the lower threshold for flow through the edge. Hence, two $k \times m$ matrices $C_{\mathrm{Max}}$ and $C_{\mathrm{Min}}$ are sufficient to summarize this information. Let $r_{a}^{j}(t)$ be the amount of a resource of dependency type $j$ produced in node $v_{a}$ at time $t$. We define $D(t)$ to be a $k \times m$ matrix over $\mathbb{Z}$ describing the amount of resources of dependency type $j$ available at the node $v_{a}$ at time $t$. It follows that the initial state of $D$ is given by $D_{a j}(0)=r_{a}^{j}(0)$. For every edge in $\mathcal{E}$ a response function 


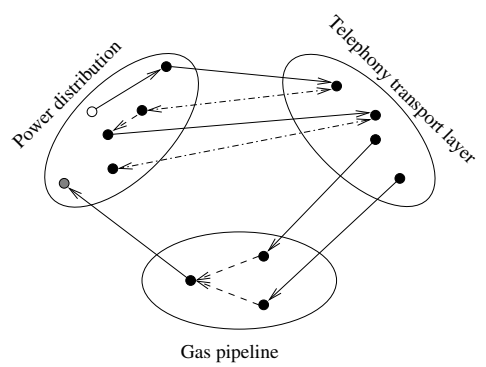

(a) Interdependencies between infrastructures. Continuous lines represent direct dependencies, dashed lines indirect dependencies, and mixed lines bidirectional dependencies

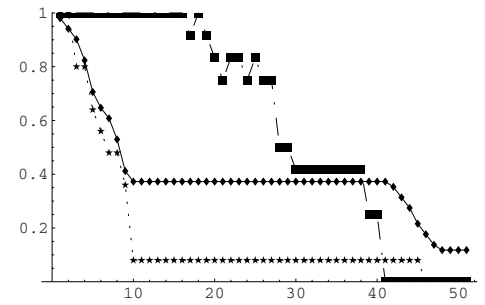

(b) Fraction of functional nodes in the power distribution network (diamond), telephony transport layer (star), and gas pipeline (block) as a function of time.

Fig. 1. Figure 1(b) shows the cascading effect of a fatal failure of the gray node of Figure 1(a) at time 0

$R_{i}^{j}\left(v_{a}, v_{b}\right): D_{a j} \times V_{a}^{j} \times N_{a}^{j} \times N_{\operatorname{Max}}\left(v_{a}, j\right) \times C_{\mathrm{Max}} \times C_{\mathrm{Min}} \rightarrow \mathbb{N}_{0}$ that determines the $i$-th flow of type $j$ between the nodes $v_{a}$ and $v_{b}$ is defined. Given the responses at time $t$, the amount of resource $j$ available in any node $v_{a}$ at time $t+1$ is given by the sum of the internally generated resources, amount resource in storage, and incoming resources to the node at time $t$. A node $v_{a}$ is said to be functional at time $t$ if it receives or generates a sufficient amount of resources to satisfy its internal needs. A metric for the level of functionality of an infrastructure is given by the sum of the functionality of the infrastructure components divided by the number of components.

Figure 1 shows the critical interdependencies between a power distribution network, a telephony transport network and a gas pipe. At time 0 in Figure 1(b) the gray power node fails. There is an immediate effect on the telecommunication and power distribution network, while the gas pipe seems to remain functional. After 25 iterations the first gas reservoirs are deprecated, and after 50 iterations the functionality of the gas pipeline drops to zero, leading to series of cascading failures in the power distribution and telecommunication networks.

\section{Conclusion}

The presented model provides a natural progression from the initial studies of large complex networks which concentrated on evaluating the robustness of attacks towards the infrastructure based on static failures 89. The flexible framework for modeling infrastructures and their interdependencies we first reported in [5], and the graph-theoretical model augmented with a set of response functions that can model both unbuffered and particularly buffered resources along with their production and consumption in a network of infrastructure components presented in [6] defines the baseline of our research. The model allows 
consideration of multiple concurrent types of interdependencies such as may arise in the provision of further infrastructure services along with simple prioritization mechanisms as may be necessary in case of some elements of the infrastructure network becomes unavailable or owing to a partitioning of the interdependency graph.

Our ongoing research focuses on one hand on extending the model to include component reliability [7] and improve time granulation, on the other hand identification of graph-theoretical and combinatorial optimization techniques (particularly as applicable to large-scale graphs) for both the identification of critical interdependencies and efficient mechanisms for increasing the robustness of such interdependent graphs. Future work includes further extensions of the model in which the response function can accommodate multiple resources being provided by each individual vertex in both discrete and continuous variables, resulting in a web of interdependencies.

\section{References}

1. Marsh, R.T., (ed.): Critical Infrastructures: Protecting America's Infrastructures. United States Government Printing Office, Washington D.C., USA, Report of the President s Commission on Critical Infrastructure Protection (1997)

2. Brömmelhörster, J., Fabry, S., Wirtz, N., (eds.): Internationale Aktivitäten zum Schutz Kritischer Infrastrukturen. Bundesamt für Sicherheit in der Informationstechnik, Bonn, Germany (2004)

3. ON Netz GmbH, E.: Bericht über den Stand der Untersuchungen zu Hergang und Ursachen der Störung des kontinentaleuropäischen Stromnetzes am Samstag, 4. November 2006 nach 22:10 Uhr. Technical report, E.ON Netz GmbH, Bayreuth, Germany (November 2006)

4. Hilt, D.: Technical Analysis of the August 14, 2003, Blackout. Technical report, North American Electric Reliability Council, Princeton, NJ, USA (July 2004)

5. Svendsen, N.K., Wolthusen, S.D.: Multigraph Dependency Models for Heterogeneous Critical Infrastructures. In: First Annual IFIP Working Group 11.10 International Conference on Critical Infrastructure Protection, Hanover, NH, USA, IFIP, (In press) Springer, Heidelberg, (March 2007)

6. Svendsen, N.K., Wolthusen, S.D.: Connectivity Models of Interdependency in Mixed-Type Critical Infrastructure Networks. Inform. Sec. Tech. Rep. 12 (In press) (March 2007)

7. Svendsen, N.K., Wolthusen, S.D.: Analysis and Statistical Properties of Critical Infrastructure Interdependency Multiflow Models. Submitted for publication (March 2007)

8. Cohen, R., Erez, K., ben-Avraham, D., Havlin, S.: Resilience of the Internet to Random Breakdowns. Physical Review Letters 85(21), 4626-4628 (2000)

9. Callaway, D.S., Newman, M.E.J., Strogatz, S.H., Watts, D.J.: Network Robustness and Fragility: Percolation on Random Graphs. Physical Review Letters 85(25), 5468-5471 (2000) 